\title{
DIFERENÇAS DE PRODUTIVIDADE DE GRÃOS ENTRE CULTIVARES INDICADOS DE MILHO, AJUSTADO PARA UM MESMO NÚMERO DE PLANTAS OU DE ESPIGAS
}

\author{
RECOMMENDED CORN CULTIVAR YIELD DIFFERENCES ADJUSTED \\ FOR THE SAME PLANT OR EAR NUMBER
}

\author{
Lindolfo Storck ${ }^{1}$ Alessandro Dal'Col Lúcio ${ }^{2}$ Thomas Newton Martin ${ }^{3}$ \\ Leandro Homrich Lorenz ${ }^{4}$ Marcelo Fernandes de Souza ${ }^{5}$
}

\section{RESUMO}

Com objetivo de caracterizar os cultivares de milho do ciclo normal, precoce e superprecoce quanto à produtividade de grãos e características morfológicas, bem como verificar se o uso da covariância da produtividade com a população de plantas ou o número de espigas aumentam a precisão dos ensaios de competição de cultivares, foram conduzidos três experimentos (um por ciclo) na área experimental do Departamento de Fitotecnia da UFSM, Santa Maria. Foi usado o delineamento em blocos ao acaso com três repetições, sendo as parcelas constituídas por duas fileiras espaçadas de $0,8 \mathrm{~m}$ com $5 \mathrm{~m}$ de comprimento, no sistema de plantio direto sobre aveia preta dessecada. Para as variáveis avaliadas foi procedida a análise da variância e covariância com teste $F$ e a aplicação do teste de Scott \& Knott para as comparações entre as médias estimadas. Concluiu-se que existe diferença de produtividade entre cultivares para um mesmo número de espigas por parcela, e que a análise da covariância torna os resultados obtidos mais precisos, reduz os valores da diferença mínima significativa, $e$ permite a apresentação de experimentos com mais qualidade.

Palavras-chave : precisão; covariância; Scott \&Knott; manejo

\section{SUMMARY}

The objectives of this study were: (i) to differentiate corn (Zea mays L.) cultivars in terms of yield and morphological traits and (ii) to verify a potential gain in experimental precision when yield covariance of plant population and/or ear number were used for statistical analysis of cultivar competition experiments. Three experiments (for middle, early-middle and early season maturity groups) were conducted at the Fitotecnia Department Experiment Station of Federal University of Santa Maria, Santa Maria, Brazil, in a non-tilling area over black oat The experimental design was a complete randomized block with three replications. The experimental unit was two rows with $0.8 \mathrm{~m}$ within-row and $5.0 \mathrm{~m}$ length. Analysis of variance and covariance was performed for all evaluated traits and Scott \& Knott was used to cluster cultivars in each maturity group. In conclusion, there are cultivar means differences adjusted for the same ear or plant number per plot and the covariance analysis improves experimental precision and reduce least significant differences.

Key words: precision; covariance; Scott \& Knott; management.

\section{INTRODUÇÃO}

A experimentação tem por objetivo delinear e executar experimentos de modo a permitir a obtenção de inferências precisas dos resultados obtidos. Para isto, o experimentador necessita utilizar técnicas refinadas com o objetivo de minimizar a variação causada por fatores não controláveis (MIRANDA FILHO, 1987). São fundamentais os princípios da repetição, casualização e controle local, onde a estimativa de uma média é tanto mais precisa quanto maior for o número de repetições utilizadas na obtenção dessa estimativa. A variância da média também diminui

\footnotetext{
${ }^{1}$ Engenheiro Agrônomo, Doutor Professor Titular, Departamento de Fitotecnia, Bolsista do CNPq, Centro de Ciências Rurais (CCR), Universidade Federal de Santa Maria (UFSM), 97105-900, Santa Maria, RS. E-mail: storck@ ccr.ufsm.br. Autor para correspondência.

${ }^{2}$ Engenheiro Agrônomo, Doutor, Professor Adjunto, Departamento Fitotecnia, CCR, UFSM.

${ }^{3}$ Acadêmico do Curso de Agronomia, UFSM, Bolsista do PIBIC.

${ }^{4}$ Acadêmico do Curso de Agronomia, UFSM, Bolsista FAPERGS.

${ }^{5}$ Engenheiro Agrônomo, Acadêmico do Curso de Pós-graduação em Agronomia, CCR/UFSM.
} Recebido para publicação em 21.08.01. Aprovado em 07.11.01 
com o aumento do número de repetições (STEEL \& TORRIE, 1960; BANZATTO \& KRONKA, 1989; GOMES, 1990). Teoricamente o erro será menor se tiver uma repetição por local, para um número fixo do total de repetições (MIRANDA FILHO, 1987).

A qualidade de um experimento é avaliada pela magnitude do erro experimental, isto é, pela variação entre unidades experimentais submetidas ao mesmo tratamento (STORCK et al., 2000). Assim, a estatística DMS (diferença mínima significativa) obtida pelo método de Tukey, quando comparada com os limites das classes previamente estabelecidos, é uma estatística adequada para o controle de qualidade (LÚCIO, 1997).

A análise dos resultados de um experimento é de boa qualidade se as pressuposições do modelo matemático estiverem sendo satisfeitas (STORCK et al., 2000). As pressuposições, para qualquer delineamento, são: aditividade dos efeitos, homogeneidade das variâncias dentro dos tratamentos, independência entre os erros e distribuição normal dos erros, as quais, quando não satisfeitas, fazem com que a análise paramétrica (teste $\mathrm{F}$ e os testes de comparações de médias) fique prejudicada, podendo levar a falsas conclusões. Se as distorções forem significativas deve-se preferir a análise não-paramétrica (CAMPOS, 1983) ou transformar os dados em uma nova escala (transformação raiz quadrada, logarítmica, arcoseno, e outras) para que os dados transformados obedeçam aproximadamente às pressuposições do modelo matemático (MARKUS, 1974).

Geralmente, como resultado de um experimento, têm-se uma variável (Y) dependente principal e variáveis (X1, X2) dependentes secundárias, denominadas de covariáveis. Considerando-se que estas covariáveis são correlacionadas entre si e com a variável principal e podem ser ou não dependentes dos tratamentos, a análise da covariância tem as suas aplicações classificadas em: a) auxiliar na interpretação dos dados experimentais; b) decompor a covariância total em componentes; c) controlar o erro e aumentar a precisão da análise de dados; d) ajustar as médias dos tratamentos em função das médias das covariáveis; e) estimar dados perdidos (STEEL \& TORRIE, 1960).

Sabe-se que a variância de um contraste entre duas médias de tratamento estimadas $\mathrm{V}(\mathrm{Z})$, para $Z=m_{i}-m_{i}$, é obtido por $V(Z)=2 \sigma^{2} / J(\mathrm{~J}=$ número de repetições), cuja estimativa é $V(Z)=$ 2. $Q M E$ / J. Para aumentar a precisão na comparação de médias de tratamentos, devemos reduzir $V(Z)$. Isso pode ser obtido aumentando-se $J$ e/ou reduzindo-se o QME. Existindo outras variáveis no experimento (covariáveis) que estejam correlacionadas com a variável principal, pode-se, através da análise da covariância, ajustar as médias da variável principal para um valor médio das covariáveis. Assim, o erro experimental da variável principal, que também é correlacionado com as covariáveis, será reduzido, podendo haver, assim, um aumento na precisão do experimento (SNEDECOR \& COCHRAN, 1967).

Desta forma, o objetivo do trabalho foi caracterizar os cultivares de milho do ciclo normal, precoce e superprecoce quanto à produtividade de grãos e características morfológicas, bem como verificar se o uso da covariância da produtividade com a população de plantas ou o número de espigas aumenta a precisão dos ensaios de competição de cultivares.

\section{MATERIAL E MÉTODOS}

Realizaram-se no Departamento de Fitotecnia da Universidade Federal de Santa Maria, três experimentos com cada um dos ciclos de maturação de milho: ciclo normal (sete cultivares e 50 mil plantas/ha), precoce (23 cultivares e $55 \mathrm{mil}$ plantas/ha) e superprecoce (20 cultivares e $65 \mathrm{mil}$ plantas/ha) na safra 1999/2000. Em cada experimento, utilizou-se o delineamento em blocos ao acaso, com três repetições. As unidades experimentais foram constituídas por duas fileiras de cinco metros de comprimento e espaçadas $0,8 \mathrm{~m}$. Devido à bordadura, desconsiderou-se os $0,5 \mathrm{~m}$ de ambas as extremidades da linha, totalizando uma área útil de $6,4 \mathrm{~m}^{2}$.

Em 24/09/99, aplicou-se 3,5 //ha de Roundup para dessecar a aveia preta e ervas daninhas existentes. Em 20/10/99, foram demarcadas e adubadas as linhas de plantio com a semeadora direta, abastecida apenas com adubo da fórmula 10-20-18, 350kg/ha. Para a semeadura, realizada no dia 21/10/99, foram colocadas manualmente duas sementes por cova, nas densidades previamente calculadas em função do ciclo das cultivares. Houve uma aplicação de herbicida Herbimix, em 28/10/99, misturado com Decis $(200 \mathrm{~m} / / \mathrm{ha})$. O desbaste das plantas excedentes nas covas foi realizado nos dias 16 e 17/10/99, e a adubação nitrogenada ocorreu na data de 30/10/99, sendo esta aplicada manualmente em cobertura, na dose de $200 \mathrm{~kg}$ de uréia/ha. Realizaram-se irrigações nos estádios mais críticos de necessidade hídrica do milho.

As variáveis coletadas no experimento foram: duração do período entre semeadura e pendoamento, sendo este determinado com $75 \%$ das plantas já apresentando pendão com pólen (média de seis plantas); estaturas de plantas (do solo à 
extremidade do pendão - média de seis plantas); altura de inserção da primeira espiga (do solo à inserção da primeira espiga - média de seis plantas); número de espigas colhidas na área útil; estande final de plantas na área útil; produtividade de grãos ajustada para $13 \%$ de umidade.

Foi procedida a análise da variância, teste F e teste de Scott \& Knott (SCOTT \& KNOTT, 1974), em nível de $5 \%$ de probabilidade de erro, para a comparação das médias. Foi calculada a estatística $D M S=100 * \Delta / m$, onde: $\Delta=q_{\alpha(n ; G L E)}$ $\sqrt{\mathrm{QME} / \mathrm{J}}$ onde $\mathrm{q}_{\alpha(\mathrm{n} ; \mathrm{GlE})}$ é o valor da tabela de Tukey para 5\% de probabilidade de erro; n é o número de cultivares e GLE (graus de liberdade do erro); QME é o quadrado médio do erro e $\mathrm{J}$ é o número de repetições.

Os dados também foram analisados usando as variáveis população final de plantas e número de espigas como covariáveis em uma análise da covariância (SNEDECOR \& COCHRAN, 1967) e, após o ajuste das estimativas das médias pela covariância, aplicou-se novamente o teste de Scott \& Knott para verificar possíveis alterações no comportamentos dessas estimativas ajustadas. As diferenças entre as estimativas da DMS, com e sem uso da covariância, foi atribuída como vantagem do uso deste procedimento.

\section{RESULTADOS E DISCUSSÃO}

Pela análise da variância realizada, para os ciclos superprecoce $(\mathrm{SP})$, precoce $(\mathrm{P})$ e normal (N) (Tabela 1), observa-se que o efeito dos cultivares mostrou-se significativo para a altura de inserção da espiga (AE), dias da semeadura à floração (DF) e produtividade de grãos (grãos t/ha) para os ciclo $\mathrm{SP}, \mathrm{P}$ e $\mathrm{N}$, ainda para as variáveis estatura da planta (AP) e número de espigas (NE), o efeito das cultivares mostrouse significativo para os ciclos $\mathrm{P}$ e $\mathrm{N}$. A única variável que não apresentou efeito significativo para as cultivares avaliadas, em nenhum dos ciclos estudados, foi o número de plantas, o que demostra que realmente os cultivares utilizados comportaram-se de maneira uniforme e próxima à população estabelecida na semeadura seguida do desbaste, ou seja, eles mantiveram um valor homogêneo no número final de plantas em todos os experimentos, estando a média da população próxima à recomendação do estande de cada ciclo, com redução final de $7,23 \%$ e 3,09\% para os ciclos SP e P, respectivamente, e acréscimo de $1,40 \%$ para o ciclo N.

O comportamento dos cultivares, inclusive naqueles em que seu efeito não foi significativo, é mostrado nas tabelas 2 e 3 . Para o ciclo P (Tabela 2), os comportamentos dos cultivares foram bem descritos pelo teste de Scott \& Knott, ou seja, naquelas variáveis onde os cultivares apresentaram efeito não significativo, pelo teste $\mathrm{F}$, o teste de comparação de médias mostrou mesma letra para todos os cultivares e, naquelas onde o efeito foi significativo, há diferenças.

Na avaliação dos ciclos SP e N (Tabela 3), houve contradições entre os dois testes aplicados na análise dos dados. Quando o teste F mostrou efeito não significativo, o teste de Scott \& Knott apresentou diferenças, para a variável estatura da planta no ciclo SP; Já quando o teste F mostrou efeito significativo, o teste de Scott \& Knott não apresentou diferenças, nas variáveis altura de inserção da espiga nos ciclos $\mathrm{SP}$ e $\mathrm{N}$ e estatura da planta e número de espigas no $\mathrm{N}$ e produtividade de grãos no SP.

Este comportamento diferenciado nos dois testes deve-se às diferenças do poder dos

Tabela 1 - Graus de liberdade (GL) e quadrados médios das variáveis estatura de plantas (AP) e altura de inserção da espiga $\mathrm{AE}$ ), número de plantas (NP) e de espigas (NE), número de dias da semeadura até a floração (DF) e produtividade de grãos de milho, causas de variação, média e diferença mínima significativa (DMS) obtida pelo teste de Tukey (5\%) expressa em percentagem da média para cultivares de ciclo superprecoce, precoce e normal. Santa Maria, 2001.

\begin{tabular}{|c|c|c|c|c|c|c|c|}
\hline $\mathrm{CV}$ & GL & $\mathrm{AP}(\mathrm{cm})$ & $\mathrm{AE}(\mathrm{cm})$ & $\begin{array}{c}\text { NP } \\
(1000 / \mathrm{ha})\end{array}$ & $\begin{array}{c}\mathrm{NE} \\
(1000 / \mathrm{ha})\end{array}$ & $\mathrm{DF}$ & $\begin{array}{l}\text { Produtividade } \\
\text { de grãos (t/ha) }\end{array}$ \\
\hline \multicolumn{8}{|c|}{ Cultivares de ciclo precoce $(\mathrm{P})$} \\
\hline Bloco & 2 & $546,9^{*}$ & $379,7 *$ & $19,3 \mathrm{~ns}$ & $34,9 \mathrm{~ns}$ & $2,8 \mathrm{~ns}$ & $1,99 \mathrm{~ns}$ \\
\hline Cultivar & 22 & $256,2 *$ & $107,2 *$ & $20,3 \mathrm{~ns}$ & $121,8^{*}$ & $11,0^{*}$ & $2,77^{*}$ \\
\hline Erro & 44 & 65,8 & 40,3 & 19,2 & 24,4 & 1,93 & 0,80 \\
\hline Média & -- & 172,4 & 106,9 & 53,3 & 50,0 & 73,5 & 6,088 \\
\hline DMS & -- & 14,8 & 18,7 & 25,8 & 31,1 & 5,9 & 46,2 \\
\hline \multicolumn{8}{|c|}{ Cultivares de ciclo superprecoce (SP) } \\
\hline Bloco & 2 & $590,7 *$ & $14,9 \mathrm{~ns}$ & $29,7 \mathrm{~ns}$ & $205,7 *$ & $7,6^{*}$ & $5,07 *$ \\
\hline Cultivar & 19 & $305,7 \mathrm{~ns}$ & $240,5^{*}$ & $68,7 \mathrm{~ns}$ & $57,4 \mathrm{~ns}$ & $19,2 *$ & $2,84 *$ \\
\hline Erro & 37 & 172,4 & 74,5 & 66,2 & 47,0 & 2,13 & 1,44 \\
\hline Média & -- & 189,4 & 113,7 & 60,3 & 57,3 & 71,0 & 7,642 \\
\hline DMS & -- & 21,6 & 23,6 & 42,0 & 37,3 & 6,4 & 48,9 \\
\hline \multicolumn{8}{|c|}{ Cultivares de ciclo normal (N) } \\
\hline Bloco & 2 & $17,0 \mathrm{~ns}$ & $243,9 \mathrm{~ns}$ & $3,1 \mathrm{~ns}$ & $69,8 \mathrm{~ns}$ & $1,5 \mathrm{~ns}$ & $2,77 *$ \\
\hline Cultivar & 6 & $476,5^{*}$ & $307,8^{*}$ & $14,8 \mathrm{~ns}$ & $178,4^{*}$ & $12,1 *$ & $4,65^{*}$ \\
\hline Erro & 12 & 132,4 & 80,9 & 9,0 & 58,9 & 0,86 & 0,60 \\
\hline Média & -- & 215,1 & 136,5 & 50,7 & 54,9 & 78,7 & 7,490 \\
\hline DMS & -- & 15,3 & 18,8 & 16,9 & 39,9 & 3,3 & 29,5 \\
\hline
\end{tabular}

* = Efeito significativo pelo teste F em nível de 5\% de probabilidade de erro; ns = não significativo. 
Tabela 2 - Médias das variáveis estatura de plantas (AP) e altura de inserção da espiga (AE), número de plantas (NP) e de espigas (NE), número de dias da semeadura até a floração (DF) e produtividade de grãos de milho para cultivares de ciclo precoce. Santa Maria, 2001 .

\begin{tabular}{lcccccc}
\hline \multicolumn{1}{c}{ Cultivar } & AP $(\mathrm{cm})$ & AE $(\mathrm{cm})$ & $\begin{array}{c}\mathrm{NP} \\
(1000 / \mathrm{ha})\end{array}$ & $\begin{array}{c}\mathrm{NE} \\
(1000 / \mathrm{ha})\end{array}$ & $\mathrm{DF}$ & $\begin{array}{c}\text { Produtividade } \\
\text { de grãos (t/ha })\end{array}$ \\
\hline AGN2003 & $180,1 \mathrm{~A}^{*}$ & $113,3 \mathrm{~A}$ & $55,73 \mathrm{~A}$ & $40,10 \mathrm{C}$ & $75,0 \mathrm{C}$ & $3,908 \mathrm{~B}$ \\
AS32 & $175,8 \mathrm{~A}$ & $115,3 \mathrm{~A}$ & $53,64 \mathrm{~A}$ & $55,72 \mathrm{~B}$ & $74,3 \mathrm{C}$ & $6,989 \mathrm{~A}$ \\
$\mathrm{AS} 523$ & $156,5 \mathrm{~B}$ & $97,8 \mathrm{~B}$ & $52,60 \mathrm{~A}$ & $55,72 \mathrm{~B}$ & $74,3 \mathrm{C}$ & $6,694 \mathrm{~A}$ \\
AS3466 & $164,9 \mathrm{~B}$ & $108,1 \mathrm{~A}$ & $54,16 \mathrm{~A}$ & $51,04 \mathrm{~B}$ & $74,3 \mathrm{C}$ & $5,401 \mathrm{~B}$ \\
AS3477 & $176,9 \mathrm{~A}$ & $109,2 \mathrm{~A}$ & $54,68 \mathrm{~A}$ & $56,77 \mathrm{~B}$ & $72,3 \mathrm{D}$ & $6,295 \mathrm{~A}$ \\
XL212 & $182,5 \mathrm{~A}$ & $104,2 \mathrm{~B}$ & $54,68 \mathrm{~A}$ & $47,91 \mathrm{~B}$ & $71,0 \mathrm{D}$ & $6,381 \mathrm{~A}$ \\
XL214 & $171,1 \mathrm{~A}$ & $103,5 \mathrm{~B}$ & $54,68 \mathrm{~A}$ & $49,47 \mathrm{~B}$ & $71,6 \mathrm{D}$ & $7,050 \mathrm{~A}$ \\
XL215 & $165,5 \mathrm{~B}$ & $101,5 \mathrm{~B}$ & $56,25 \mathrm{~A}$ & $53,12 \mathrm{~B}$ & $71,0 \mathrm{D}$ & $6,513 \mathrm{~A}$ \\
XL340 & $180,3 \mathrm{~A}$ & $107,6 \mathrm{~A}$ & $53,12 \mathrm{~A}$ & $36,45 \mathrm{C}$ & $71,0 \mathrm{D}$ & $4,679 \mathrm{~B}$ \\
XL344 & $171,9 \mathrm{~A}$ & $102,7 \mathrm{~B}$ & $54,68 \mathrm{~A}$ & $44,27 \mathrm{C}$ & $71,6 \mathrm{D}$ & $4,814 \mathrm{~B}$ \\
XL345 & $176,1 \mathrm{~A}$ & $105,3 \mathrm{~B}$ & $46,35 \mathrm{~A}$ & $42,70 \mathrm{C}$ & $73,6 \mathrm{C}$ & $5,656 \mathrm{~B}$ \\
CO32 & $167,9 \mathrm{~B}$ & $111,1 \mathrm{~A}$ & $56,77 \mathrm{~A}$ & $54,68 \mathrm{~B}$ & $73,6 \mathrm{C}$ & $6,595 \mathrm{~A}$ \\
D1000 & $170,5 \mathrm{~A}$ & $112,9 \mathrm{~A}$ & $56,77 \mathrm{~A}$ & $66,66 \mathrm{~A}$ & $75,6 \mathrm{~B}$ & $7,181 \mathrm{~A}$ \\
TORK & $154,3 \mathrm{~B}$ & $104,6 \mathrm{~B}$ & $50,52 \mathrm{~A}$ & $49,47 \mathrm{~B}$ & $75,0 \mathrm{C}$ & $6,991 \mathrm{~A}$ \\
PREMIUM & $171,5 \mathrm{~A}$ & $109,8 \mathrm{~A}$ & $50,52 \mathrm{~A}$ & $52,60 \mathrm{~B}$ & $76,3 \mathrm{~B}$ & $7,377 \mathrm{~A}$ \\
AVANT & $155,6 \mathrm{~B}$ & $95,2 \mathrm{~B}$ & $52,08 \mathrm{~A}$ & $45,31 \mathrm{C}$ & $72,3 \mathrm{D}$ & $5,786 \mathrm{~B}$ \\
G800 & $182,7 \mathrm{~A}$ & $114,4 \mathrm{~A}$ & $54,68 \mathrm{~A}$ & $51,04 \mathrm{~B}$ & $71,6 \mathrm{D}$ & $6,832 \mathrm{~A}$ \\
G8447G & $192,7 \mathrm{~A}$ & $117,8 \mathrm{~A}$ & $52,60 \mathrm{~A}$ & $43,22 \mathrm{C}$ & $73,0 \mathrm{D}$ & $5,248 \mathrm{~B}$ \\
SHS5060 & $167,8 \mathrm{~B}$ & $100,8 \mathrm{~B}$ & $53,64 \mathrm{~A}$ & $48,95 \mathrm{~B}$ & $73,0 \mathrm{D}$ & $5,874 \mathrm{~B}$ \\
C435 & $174,1 \mathrm{~A}$ & $108,7 \mathrm{~A}$ & $51,04 \mathrm{~A}$ & $49,47 \mathrm{~B}$ & $78,3 \mathrm{~A}$ & $5,313 \mathrm{~B}$ \\
C444 & $172,3 \mathrm{~A}$ & $101,1 \mathrm{~B}$ & $51,04 \mathrm{~A}$ & $53,12 \mathrm{~B}$ & $75,6 \mathrm{~B}$ & $5,935 \mathrm{~B}$ \\
Z8474 & $172,2 \mathrm{~A}$ & $101,2 \mathrm{~B}$ & $49,47 \mathrm{~A}$ & $48,43 \mathrm{~B}$ & $72,3 \mathrm{D}$ & $7,453 \mathrm{~A}$ \\
Z8486 & $181,1 \mathrm{~A}$ & $112,4 \mathrm{~A}$ & $56,25 \mathrm{~A}$ & $53,12 \mathrm{~B}$ & $73,6 \mathrm{C}$ & $5,059 \mathrm{~B}$ \\
& & & & & & \\
\hline
\end{tabular}

* Cultivares com médias não ligadas por mesma letra diferem pelo teste de Scott \& Knott em nível de $5 \%$ de probabilidade de erro.

mesmos, sendo recomendada uma maior prioridade ao resultado apresentado pelo teste F. Isso significa que, quando este teste mostra efeito significativo, não é necessário aplicar nenhum outro teste complementar de comparação de médias para a mesma variável. Quando o teste F apresentar efeito significativo para os tratamentos, aplica-se então um teste de comparação de médias para discriminar as diferenças existentes, mas esse comportamento não foi observado em algumas variáveis, como mencionado anteriormente, principalmente para a variável produtividade de grãos que, dentro de um experimento de avaliação de cultivares, é a principal variável estudada, definindo as recomendações de cultivo comercial.

Para solucionar este problema, escolheram-se as variáveis número de plantas (NP) e número de espigas (NE) que, apesar de não apresentarem efeito significativo para os cultivares no teste $F$, são aquelas que possuem maior grau de interferência na produtividade final de grãos, para serem utilizadas como covariáveis na correção dos valores das médias de produtividade dos cultivares avaliados.

Com a análise da covariância (Tabela 4), utilizando-se as duas covariáveis definidas, o efeito dos cultivares permanece significativo para todos os ciclos e com a covariável NP, há um efeito significativo do uso da covariância para o ciclo SP, que é o ciclo que apresentou incompatibilidade entre os resultados dos testes $\mathrm{F}$ e de Scott \& Knott. Já com a covariável NE, além de apresentar efeito significativo para o uso da covariância, em todos os ciclos, há uma redução substancial nos valores da DMS, de 25,97, 13,63 e 19,32\% para os ciclos $\mathrm{SP}, \mathrm{P}$ e $\mathrm{N}$, respectivamente, comprovando que, com a análise da covariância, além de se obter maior exatidão, há maior precisão no que se refere à redução do erro experimental.

Para comprovar que a análise da covariância é uma forma de maximizar a precisão e a exatidão de um experimento, aplicou-se novamente o teste de Scott \& Knott nas médias ajustadas dos cultivares, para as duas covariáveis, como mostram as tabelas 5 e 6 .

Com a covariável número de plantas (NP), o comportamento dos cultivares de milho, com relação à média da produtividade, não se alterou para os ciclos $\mathrm{N}$ e $\mathrm{P}$, apesar destas médias sofrerem um ajuste, ou seja, não houve uma mudança de grupo ao se realizar o ajuste pela covariável NP. Os cultivares que haviam sido classificados no melhor grupo, com as médias não ajustadas, mantiveram-se neste mesmo grupo, com as médias ajustadas, apresentando o mesmo comportamento para os cultivares classificados no pior grupo.

Já com a covariável número de espigas (NE), houve mudanças de grupos para todos os ciclos, e a maior mudança apresentou-se para o ciclo superprecoce. A mudança de grupo ocorreu nos dois sentidos, ou seja, cultivares classificados no grupo superior passaram para um inferior e vice-versa. Observou-se que, no ciclo precoce, dois cultivares (AS3477 e D1000) tiveram classificação, com as médias ajustadas, no grupo inferior e outros dois cultivares (XL345 e Avant) passaram a ser classificados no grupo superior. Pela tabela 2, observa-se que os cultivares que tiveram sua nova média da produtividade de grãos classificada no grupo superior apresentavam um número de espigas classificados no grupo inferior, com uma relação de menos de 1:1 no número de plantas e número de 
Tabela 3 - Médias das variáveis estatura de plantas (AP) e altura de inserção da espiga (AE), número de plantas (NP) e de espigas (NE), número de dias da semeadura até a floração (DF) e produtividade de grãos de milho para cultivares de ciclo superprecoce e normal. Santa Maria, 2001.

\begin{tabular}{|c|c|c|c|c|c|c|}
\hline Cultivar & $\mathrm{AP}(\mathrm{cm})$ & $\mathrm{AE}(\mathrm{cm})$ & $\begin{array}{c}\text { NP } \\
(1000 / \text { ha })\end{array}$ & $\begin{array}{c}\mathrm{NE} \\
(1000 / \mathrm{ha})\end{array}$ & $\mathrm{DF}$ & $\begin{array}{l}\text { Produtividade } \\
\text { de grãos (t/ha) }\end{array}$ \\
\hline \multicolumn{7}{|c|}{ Cultivares de ciclo superprecoce $(\mathrm{P})$} \\
\hline AGN2012 & $191,1 \mathrm{~B} *$ & $116,3 \mathrm{~A}$ & $63,02 \mathrm{~A}$ & $54,16 \mathrm{~A}$ & $70,3 \mathrm{C}$ & 7,293 A \\
\hline AGN3050 & $181,7 \mathrm{~B}$ & $114,6 \mathrm{~A}$ & $60,94 \mathrm{~A}$ & $59,89 \mathrm{~A}$ & $71,0 \mathrm{~B}$ & $8,187 \mathrm{~A}$ \\
\hline AGN3060 & 192,7 B & $116,6 \mathrm{~A}$ & $59,89 \mathrm{~A}$ & $50,52 \mathrm{~A}$ & $74,3 \mathrm{~A}$ & $6,016 \mathrm{~A}$ \\
\hline AGN3100 & $195,5 \mathrm{~B}$ & $120,2 \mathrm{~A}$ & $67,71 \mathrm{~A}$ & $66,14 \mathrm{~A}$ & $73,0 \mathrm{~A}$ & $6,738 \mathrm{~A}$ \\
\hline AGN3150 & $182,2 \mathrm{~B}$ & $118,1 \mathrm{~A}$ & $60,41 \mathrm{~A}$ & $55,72 \mathrm{~A}$ & $72,3 \mathrm{~B}$ & $5,583 \mathrm{~A}$ \\
\hline AGN3180 & $183,6 \mathrm{~B}$ & $119,6 \mathrm{~A}$ & $59,89 \mathrm{~A}$ & $59,37 \mathrm{~A}$ & $73,7 \mathrm{~A}$ & $7,614 \mathrm{~A}$ \\
\hline AS3601 & $177,6 \mathrm{~B}$ & $115,4 \mathrm{~A}$ & $52,08 \mathrm{~A}$ & $53,64 \mathrm{~A}$ & $73,0 \mathrm{~A}$ & $7,005 \mathrm{~A}$ \\
\hline XL205 & $187,2 \mathrm{~B}$ & $92,9 \mathrm{~A}$ & $59,37 \mathrm{~A}$ & $57,81 \mathrm{~A}$ & $65,6 \mathrm{D}$ & $8,573 \mathrm{~A}$ \\
\hline XL220 & $179,0 \mathrm{~B}$ & $121,1 \mathrm{~A}$ & $56,77 \mathrm{~A}$ & $48,95 \mathrm{~A}$ & $71,7 \mathrm{~B}$ & $8,802 \mathrm{~A}$ \\
\hline CD3211 & $215,0 \mathrm{~A}$ & $142,4 \mathrm{~A}$ & $63,28 \mathrm{~A}$ & $57,81 \mathrm{~A}$ & $75,0 \mathrm{~A}$ & $6,949 \mathrm{~A}$ \\
\hline P32R 21 & $213,1 \mathrm{~A}$ & $106,3 \mathrm{~A}$ & $59,37 \mathrm{~A}$ & $58,85 \mathrm{~A}$ & $66,0 \mathrm{D}$ & $9,593 \mathrm{~A}$ \\
\hline SHS4050 & $186,2 \mathrm{~B}$ & $115,1 \mathrm{~A}$ & $64,58 \mathrm{~A}$ & $55,20 \mathrm{~A}$ & $72,3 \mathrm{~B}$ & $7,428 \mathrm{~A}$ \\
\hline SHS5050 & 182,2 B & $108,5 \mathrm{~A}$ & $63,54 \mathrm{~A}$ & $59,89 \mathrm{~A}$ & $69,0 \mathrm{C}$ & $7,925 \mathrm{~A}$ \\
\hline SHS5070 & $186,8 \mathrm{~B}$ & $120,8 \mathrm{~A}$ & $63,02 \mathrm{~A}$ & $56,25 \mathrm{~A}$ & $71,0 \mathrm{~B}$ & $7,640 \mathrm{~A}$ \\
\hline AG6016 & $181,4 \mathrm{~B}$ & $109,4 \mathrm{~A}$ & $64,06 \mathrm{~A}$ & $61,97 \mathrm{~A}$ & $69,6 \mathrm{C}$ & $8,827 \mathrm{~A}$ \\
\hline AG6018 & $188,4 \mathrm{~B}$ & $104,4 \mathrm{~A}$ & $55,73 \mathrm{~A}$ & $57,29 \mathrm{~A}$ & $69,6 \mathrm{C}$ & $7,967 \mathrm{~A}$ \\
\hline C806 & $205,2 \mathrm{~A}$ & $113,4 \mathrm{~A}$ & $48,96 \mathrm{~A}$ & $58,33 \mathrm{~A}$ & $69,0 \mathrm{C}$ & $8,566 \mathrm{~A}$ \\
\hline $\mathrm{C} 855$ & $189,3 \mathrm{~B}$ & $115,7 \mathrm{~A}$ & $66,14 \mathrm{~A}$ & $64,58 \mathrm{~A}$ & $74,3 \mathrm{~A}$ & $7,400 \mathrm{~A}$ \\
\hline $\mathrm{C} 801$ & $184,1 \mathrm{~B}$ & $107,5 \mathrm{~A}$ & $64,58 \mathrm{~A}$ & $58,85 \mathrm{~A}$ & $69,0 \mathrm{C}$ & $7,280 \mathrm{~A}$ \\
\hline C909 & $193,1 \mathrm{~B}$ & $104,8 \mathrm{~A}$ & $54,68 \mathrm{~A}$ & $51,04 \mathrm{~A}$ & $71,6 \mathrm{~B}$ & $7,219 \mathrm{~A}$ \\
\hline \multicolumn{7}{|c|}{ Cultivares de ciclo normal (N) } \\
\hline XL 370 & $218,5 \mathrm{~A}$ & $139,5 \mathrm{~A}$ & $48,43 \mathrm{~A}$ & $59,37 \mathrm{~A}$ & $77,0 \mathrm{~B}$ & $8,417 \mathrm{~A}$ \\
\hline XL 660 & $198,9 \mathrm{~A}$ & $132,1 \mathrm{~A}$ & $51,56 \mathrm{~A}$ & $60,41 \mathrm{~A}$ & $77,6 \mathrm{~B}$ & $8,055 \mathrm{~A}$ \\
\hline B 670 & $238,4 \mathrm{~A}$ & $146,4 \mathrm{~A}$ & $49,47 \mathrm{~A}$ & $66,66 \mathrm{~A}$ & $80,6 \mathrm{~A}$ & $8,979 \mathrm{~A}$ \\
\hline C 333 B & $215,5 \mathrm{~A}$ & $133,3 \mathrm{~A}$ & $50,52 \mathrm{~A}$ & $53,12 \mathrm{~A}$ & $77,0 \mathrm{~B}$ & $6,650 \mathrm{~B}$ \\
\hline OC 705 & $207,9 \mathrm{~A}$ & $118,3 \mathrm{~A}$ & $55,20 \mathrm{~A}$ & $44,27 \mathrm{~A}$ & $81,0 \mathrm{~A}$ & $5,217 \mathrm{C}$ \\
\hline P 3232 & $206,8 \mathrm{~A}$ & $136,8 \mathrm{~A}$ & $50,00 \mathrm{~A}$ & $47,91 \mathrm{~A}$ & $77,0 \mathrm{~B}$ & $7,446 \mathrm{~A}$ \\
\hline AG 1051 & $219,4 \mathrm{~A}$ & $148,6 \mathrm{~A}$ & $49,47 \mathrm{~A}$ & $53,12 \mathrm{~A}$ & $81,0 \mathrm{~A}$ & $7,666 \mathrm{~A}$ \\
\hline
\end{tabular}

* Cultivares com médias não ligadas por mesma letra diferem pelo teste de Scott \& Knott em nível

Espigas, e aqueles que tiveram a média ajustada classificada no grupo inferior apresentavam os maiores valores do NE, com uma relação maior que 1:1 no NP:NE.

Para o ciclo superprecoce (Tabela 6), com o uso da covariável NE, os cultivares passaram a ser classificados em grupos distintos, resultado não apresentado sem o ajuste das médias, mantendo o mesmo sentido de resposta em relação ao número de espigas de cada cultivar. Isso quer dizer que cultivares apresentando NE alto tiveram o valor da média de produtividade de grãos reduzido, como, por exemplo, o cultivar AGN3060 e com NE baixo, a produtividade de grãos sofreu um acréscimo, como, por exemplo, o cultivar XL220. No ciclo normal (Tabela 6) apenas o cultivar OC705 apresentou um acréscimo no valor da média de produtividade de grãos, guardando o mesmo comportamento discutido anteriormente, pois este possui o menor número de espigas e a menor relação NP:NE dos cultivares avaliados.

O ajuste realizado pelo uso da

Tabela 4 - Graus de liberdade (GL) e quadrados médios (QM) da análise da covariância da produtividade de grãos de milho (t/ha) em função do número de plantas e de espigas, média e diferença mínima significativa (DMS) obtida pelo teste de Tukey (5\%) expressa em percentagem da média para cultivares de ciclo superprecoce, precoce e normal. Santa Maria, 2001.

\begin{tabular}{lcccccc}
\hline \multirow{2}{*}{ Parâmetro } & \multicolumn{2}{l}{ Superprecoce (SP) } & \multicolumn{2}{l}{ Precoce (P) } & \multicolumn{2}{l}{ Normal (N) } \\
\cline { 2 - 7 } & GL & QM & GL & QM & GL & QM \\
\cline { 2 - 7 } & \multicolumn{7}{c}{ Cov $=$ número de plantas (milhares / ha) } \\
Bloco & 2 & $4,87^{*}$ & 2 & $2,53 \mathrm{~ns}$ & 2 & $2,92^{*}$ \\
Cultivar & 19 & $3,13^{*}$ & 22 & $2,80^{*}$ & 6 & $4,00^{*}$ \\
Cov & 1 & $7,17^{*}$ & 1 & $0,69 \mathrm{~ns}$ & 1 & $0,97 \mathrm{~ns}$ \\
Erro & 36 & 1,283 & 43 & 0,800 & 11 & 0,568 \\
Média & -- & 7,642 & -- & 6,088 & -- & 7,490 \\
DMS & -- & 46,3 & -- & 46,2 & -- & 28,7 \\
& Cov & número de espigas (milhares / ha) & \\
Bloco & 2 & $0,46 \mathrm{~ns}$ & 2 & $3,62^{*}$ & 2 & $1,81^{*}$ \\
Cultivar & 19 & $3,01^{*}$ & 22 & $1,62^{*}$ & 6 & $1,51^{*}$ \\
Cov & 1 & $25,12^{*}$ & 1 & $9,42^{*}$ & 1 & $2,91^{*}$ \\
Erro & 36 & 0,785 & 43 & 0,597 & 11 & 0,392 \\
Média & -- & 7,642 & - & 6,088 & -- & 7,490 \\
DMS & -- & 36,2 & - & 39,9 & -- & 23,8 \\
& \multicolumn{7}{c}{36,9} \\
\hline
\end{tabular}

* = Efeito significativo pelo teste $\mathrm{F}$ em nível de $5 \%$ de probabilidade de erro; ns = não significativo. covariância ficou evidenciado e mostrou que, ao se aplicar tal análise, grandes diferenças entre médias de produtividade de grãos que não se apresentavam significativas, passaram a ser considerada significativas. Com esse uso também a classificação realizada pelo teste de Scott \& Knott, após o ajuste das médias, ficou mais precisa e confiável, podendo-se retirar os efeitos de outras variáveis observadas no experimento que interferem diretamente na produtividade final de grãos. As diferenças entre os cultivares, usando médias ajustadas pelas covariáveis, se devem a fatores genéticos não devidos a estas covariáveis e, portanto, o número de espigas e/ou número de plantas não são os únicos fatores determinantes da produtividade do milho.

\section{CONCLUSÕES}

O número de espigas pode ser utilizado como covariável para correção das médias da variável produtividade de grãos. A análise da 
Tabela 5 - Médias da produtividade de grãos de milho ajustada para as covariáveis número de plantas e número de espigas para cultivares de ciclo precoce. Santa Maria, 2001.

\begin{tabular}{lcc}
\hline Cultivar & Número de plantas & Número de espigas \\
\hline AGN2003 & $3,839 \mathrm{~B} *$ & $4,832 \mathrm{~B}$ \\
AS32 & $6,979 \mathrm{~A}$ & $6,451 \mathrm{~A}$ \\
AS523 & $6,715 \mathrm{~A}$ & $6,156 \mathrm{~A}$ \\
AS3466 & $5,376 \mathrm{~B}$ & $5,301 \mathrm{~B}$ \\
AS3477 & $6,255 \mathrm{~A}$ & $5,659 \mathrm{~B}$ \\
XL212 & $6,342 \mathrm{~A}$ & $6,574 \mathrm{~A}$ \\
XL214 & $7,011 \mathrm{~A}$ & $7,097 \mathrm{~A}$ \\
XL215 & $6,428 \mathrm{~A}$ & $6,218 \mathrm{~A}$ \\
XL340 & $4,685 \mathrm{~B}$ & $5,944 \mathrm{~B}$ \\
XL344 & $4,775 \mathrm{~B}$ & $5,348 \mathrm{~B}$ \\
XL345 & $5,855 \mathrm{~B}$ & $6,336 \mathrm{~A}$ \\
CO32 & $6,496 \mathrm{~A}$ & $6,155 \mathrm{~A}$ \\
D1000 & $7,081 \mathrm{~A}$ & $5,619 \mathrm{~B}$ \\
TORK & $7,071 \mathrm{~A}$ & $7,037 \mathrm{~A}$ \\
PREMIUM & $7,456 \mathrm{~A}$ & $7,131 \mathrm{~A}$ \\
AVANT & $5,821 \mathrm{~B}$ & $6,222 \mathrm{~A}$ \\
G800 & $6,732 \mathrm{~A}$ \\
G8447G & $6,792 \mathrm{~A}$ & $5,880 \mathrm{~B}$ \\
SHS5060 & $5,268 \mathrm{~B}$ & $5,969 \mathrm{~B}$ \\
C435 & $5,864 \mathrm{~B}$ & $5,359 \mathrm{~B}$ \\
C444 & $5,378 \mathrm{~B}$ & $5,641 \mathrm{~B}$ \\
Z8474 & $6,001 \mathrm{~B}$ & $7,597 \mathrm{~A}$ \\
Z8486 & $7,563 \mathrm{~A}$ & $4,764 \mathrm{~B}$ \\
\hline & $4,974 \mathrm{~B}$ &
\end{tabular}

* Cultivares com médias não ligadas por mesma letra diferem pelo teste de Scott \& Knott em nível de 5\% de probabilidade de erro.

covariância torna os resultados obtidos mais precisos, reduz os valores da diferença mínima significativa, e permite apresentação mais qualificada dos experimentos.

\section{REFERÊNCIAS BIBLIOGRÁFICAS}

BANZATTO, D.A., KRONKA, S.N. Experimentação agrícola. Jaboticabal : FUNEP, 1989. 247p.

CAMPOS, H. Estatística experimental não-paramétrica. 4.ed. Piracicaba : Departamento de Matemática e Estatística ESALQ, 1983. 349p.

GOMES, F.P. Curso de estatística experimental. 13.ed. Piracicaba : Nobel, 1990. 468p.

LÚCIO, A.D. Parâmetros da precisão experimental das principais culturas anuais do Estado do Rio Grande do Sul. Santa Maria - RS, 1997. 64p. Dissertação (Mestrado em Agronomia) - Curso de Pós-graduação em Agronomia, Centro de Ciências Rurais, Universidade Federal de Santa Maria, 1997.

MARKUS, M. Elementos de estatística aplicada Porto Alegre: UFRGS / Departamento de Estatística, 1974. 329p.
Tabela 6 - Médias da produtividade de grãos de milho ajustada para as covariáveis número de plantas e número de espigas para cultivares de ciclo super precoce e normal. Santa Maria, 2001.

\begin{tabular}{|c|c|c|}
\hline Cultivar & Número de plantas & Número de espigas \\
\hline \multicolumn{3}{|c|}{ Cultivares de ciclo super precose (SP) } \\
\hline AGN2012 & 7,149 B* & 7,671 B \\
\hline AGN3050 & $8,156 \mathrm{~A}$ & $7,876 \mathrm{~B}$ \\
\hline AGN3060 & $6,041 \mathrm{~B}$ & $6,833 \mathrm{C}$ \\
\hline AGN3100 & $6,340 \mathrm{~B}$ & $5,676 \mathrm{C}$ \\
\hline AGN3150 & $5,579 \mathrm{~B}$ & $5,773 \mathrm{C}$ \\
\hline AGN3180 & $7,639 \mathrm{~B}$ & $7,366 \mathrm{~B}$ \\
\hline AS3601 & $7,453 \mathrm{~B}$ & $7,445 \mathrm{~B}$ \\
\hline XL205 & $8,626 \mathrm{~A}$ & $8,513 \mathrm{~B}$ \\
\hline XL220 & $8,995 \mathrm{~A}$ & $9,805 \mathrm{~A}$ \\
\hline CD3211 & $6,543 \mathrm{~B}$ & $6,795 \mathrm{C}$ \\
\hline P32R21 & $9,646 \mathrm{~A}$ & $9,407 \mathrm{~A}$ \\
\hline SHS4050 & $7,199 \mathrm{~B}$ & $7,680 \mathrm{~B}$ \\
\hline SHS5050 & $7,753 \mathrm{~B}$ & 7,614 B \\
\hline SHS5070 & $7,495 \mathrm{~B}$ & $7,767 \mathrm{~B}$ \\
\hline AG6016 & $8,627 \mathrm{~A}$ & $8,266 \mathrm{~B}$ \\
\hline AG6018 & $8,217 \mathrm{~A}$ & $7,969 \mathrm{~B}$ \\
\hline C806 & $9,183 \mathrm{~A}$ & $8,443 \mathrm{~B}$ \\
\hline $\mathrm{C} 855$ & 7,087 B & $6,526 \mathrm{C}$ \\
\hline C801 & 7,051 B & 7,094 C \\
\hline C909 & $7,526 \mathrm{~B}$ & $7,972 \mathrm{~B}$ \\
\hline \multicolumn{3}{|c|}{ Cultivares de ciclo normal (N) } \\
\hline XL 370 & $8,629 \mathrm{~A}$ & $8,136 \mathrm{~A}$ \\
\hline XL 660 & $7,970 \mathrm{~A}$ & 7,707 A \\
\hline В 670 & $9,092 \mathrm{~A}$ & $8,230 \mathrm{~A}$ \\
\hline C 333 B & $6,664 \mathrm{~B}$ & $6,769 \mathrm{~B}$ \\
\hline OC 705 & $4,786 \mathrm{C}$ & 5,904 B \\
\hline P 3232 & $7,510 \mathrm{~A}$ & $7,899 \mathrm{~A}$ \\
\hline AG 1051 & $7,779 \mathrm{~A}$ & $7,786 \mathrm{~A}$ \\
\hline
\end{tabular}

* Cultivares com médias não ligadas por mesma letra diferem pelo teste de Scott \& Knott em nível de 5\% de probabilidade de erro.

MIRANDA FILHO, J.B. Princípios de experimentação e análise estatística. In.: PATERNIANI, E., VIEGAS, G.P. Melhoramento e produção do milho. 2.ed. Campinas : Fundação Cargill, 1987. V.2, p.765-795.

SCOTT, A.J., KNOTT, M.A. A cluster analysis method for grouping means in the analysis of variance. Biometrics, Raleigh, v.30, n.3, p.507-512, 1974.

SNEDECOR, G.W., COCHRAN, W.G. Statistical methods. 6.ed. Ames : Iowa State University, 1967. 593p.

STEEL, R.G.D., TORRIE, J.H. Principles and procedures of statistics. New York: McGraw Hill, 1960. 481p.

STORCK, L., GARCIA, D.C., LOPES, S.J., et al. Experimentação vegetal. Santa Maria : UFSM, 2000. 198p. 